\title{
Molecular ISM and Star Formation Efficiency in the Central Kpc
}

\author{
Shardha Jogee \\ Division of Physics, Mathematics, and Astronomy, MS 105-24, \\ California Institute of Technology, Pasadena, CA 91125
}

\section{Introduction}

Why do the circumnuclear (inner 1-2 kpc) regions of some spirals develop starbursts, which are intense short-lived $(\mathrm{t} \ll 1 \mathrm{Gyr}$ ) episodes of star formation characterized by a high star formation rate per unit mass of molecular gas $\left(\mathrm{SFR} / M_{\mathrm{H} 2}\right.$ ), which I refer to as the star formation efficiency (SFE), while others with comparable gas content become non-starbursts? I address this question and other issues on circumnuclear evolution using high resolution (2" or 100-200 pc) $\mathrm{CO}(\mathrm{J}=1->0)$ observations from the Owens Valley Radio Observatory, optical and NIR images, along with radio continuum (RC) and $\mathrm{Br} \gamma$ data. The sample galaxies are all nearby, moderately inclined, and include the brightest nearby starbursts comparable to M82 and control non-starbursts. Detailed results are in Jogee (1999) and Jogee, Kenney, \& Scoville 2000 (in prep.)

\subsection{The Circumnuclear Molecular Gas}

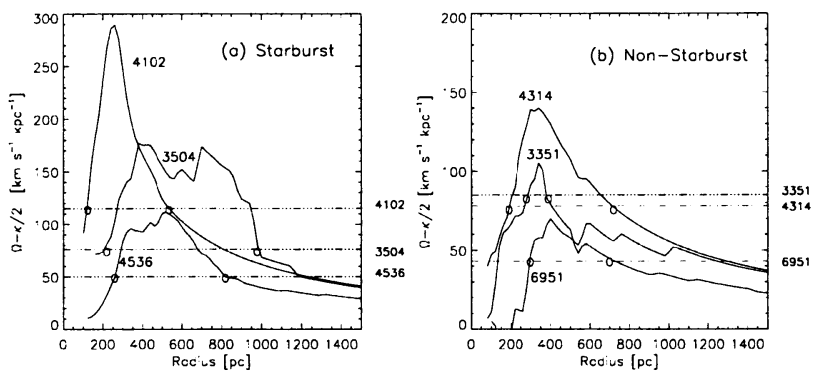

Figure 1. Inner Lindblad resonances: $[\Omega-\kappa / 2]$ is plotted against radius. The bar pattern speed $\Omega_{\mathrm{p}}$ is drawn as horizontal lines.

What is the circumnuclear gas content, morphology, and how do they relate to the properties of the barred potential? (i) The sample galaxies have developed large molecular gas reservoirs of several $10^{8}-10^{9} M_{\odot}$ in the inner kpc radius, assuming a standard $\mathrm{CO}-\mathrm{to}_{\mathrm{H}} \mathrm{H}_{2}$ conversion factor. The sample properties are consistent with the idea that a spontaneously or tidally induced bar, and minor mergers/interactions can help to drive gas towards the inner kpc. All sample galaxies show a large-scale stellar bar/oval whose position angle is marked on Fig. 2, and all galaxies, except for NGC 6951, show evidence for recent tidal interactions/mergers with mass ratios ranging from minor (1:10) to intermediate. The presence of a large-scale bar in starbursts and non-starbursts 


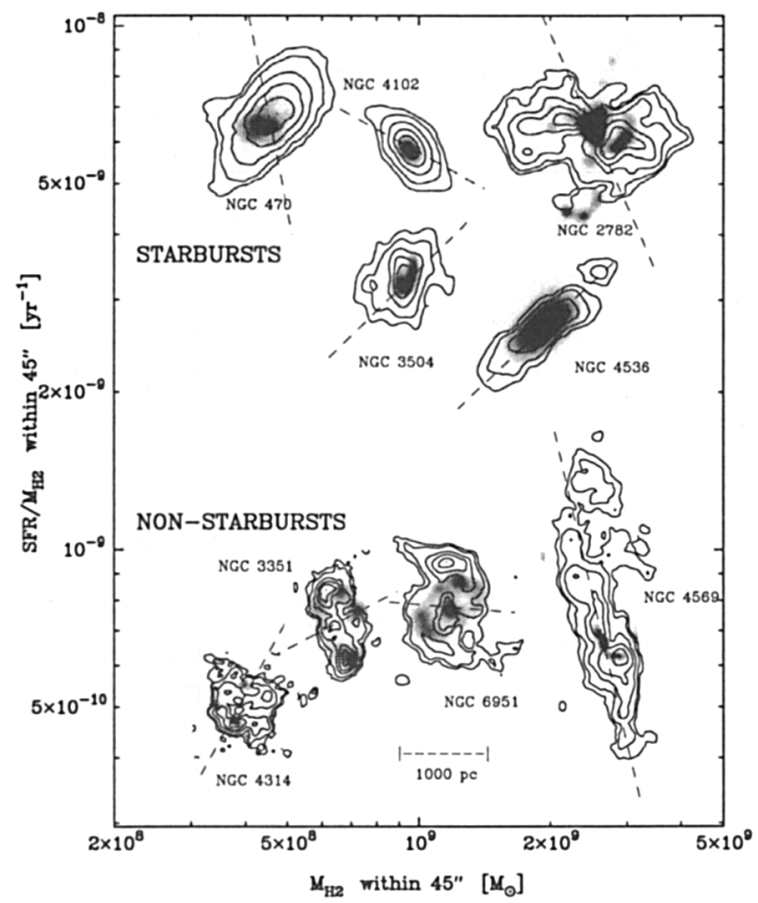

Figure 2. In the SFR $/ M_{\mathrm{H} 2}$ vs $M_{\mathrm{H} 2}$ plane, the $\mathrm{CO}$ intensity (contours) is overlaid on the star formation (greyscale), as traced by RC and by $\mathrm{H} \alpha$. The dotted line is the P.A. of the large-scale stellar bar/oval.

alike suggests that the starburst lifetime ( $\leq$ few $\times 10^{8}$ years.) is short with respect to the timescale for bar dissolution. Thus, within a given barred potential, star formation can change from an inefficient pre-burst phase in the early stages of bar-driven inflow, to a circumnuclear starburst, to a post-starburst. (ii) In NGC 4569 , the gas extends out to a large $(2 \mathrm{kpc})$ radius, at a similar P.A. as the large-scale stellar bar (Fig. 2), and shows complex non-circular motions. The warped disk, asymmetric bar and CO properties in NGC 4569 suggest it is in the early stages of bar-driven/tidally-driven inflow of gas towards the inner kpc (Jogee 1999). (iii) In the other galaxies, the gas distribution is less extended, and has variety of morphologies (Fig. 2) including relatively axisymmetric annuli or disks (starbursts NGC 4102, NGC 3504, NGC 4536, and non-starbursts NGC 4314), elongated double-peaked and spiral morphologies (starburst NGC 2782 and non-starbursts NGC 3351 and NGC 6951). The gas is often concentrated inside the outer inner Lindblad resonance (ILR) of the large-scale bar. As shown in Fig. 1, both starbursts and non-starbursts host ILRs. The bar pattern speed $\Omega_{\mathrm{p}}$ ranges from 40 to $115 \mathrm{~km} \mathrm{~s}^{-1} \mathrm{kpc}^{-1}$, the radius of the outer ILR is typically $>500 \mathrm{pc}$, and the radius of the inner IILR $<300 \mathrm{pc}$.

The marked difference between molecular gas in the inner kpc and the outer disk (see Table 1) has important implications for the circumnuclear region: (i) The high molecular gas density (several $100-1000 \mathrm{M}_{\odot} \mathrm{pc}^{-2}$ ) and mass fraction (10 to $30 \%$ ) will lead to enhanced self-gravity and clumpiness of the gas (e.g., 
Table 1. Molecular Gas Properties In The Circumnuclear Region

\begin{tabular}{lccc}
\hline Quantities & $\begin{array}{c}\text { Outer Disk } \\
\text { of Sa-Sc }\end{array}$ & $\begin{array}{c}\text { Inner } \mathrm{r}=500 \mathrm{pc} \\
\text { of sample galaxies }\end{array}$ & $\begin{array}{c}\text { Inner } \mathrm{r}=500 \mathrm{pc} \\
\text { of Arp } 220\end{array}$ \\
\hline (1) $M_{\text {gas-m }}\left[M_{\odot}\right]$ & $\leq$ few $\times 10^{9}$ & Few $\times\left(10^{8}-10^{9}\right)$ & $3 \times 10^{9}$ \\
(2) $\mathrm{M}_{\text {gas }} / \mathrm{M}_{\mathrm{dyn}}[\%]$ & $<5$ & 10 to 30 & 40 to 80 \\
(3) $\mathrm{SFR}\left[M_{\odot} \mathrm{yr}^{-1}\right]$ & - & $0.1-11$ & $>100$ \\
(4) $\Sigma_{\text {gas,m }}\left[M_{\odot} \mathrm{pc}^{-2}\right]$ & $1-100$ & $500-3500$ & $4 \times 10^{4}$ \\
(5) $\sigma\left[\mathrm{km} \mathrm{s}^{-1}\right]$ & $6-10$ & $10-40$ & 90 \\
(6) $\kappa\left[\mathrm{km} \mathrm{s}^{-1} \mathrm{kpc}^{-1}\right]$ & $<100$ & $800-3000$ & $>1000$ \\
(7) $\Sigma_{\mathrm{crit}}\left[M_{\odot} \mathrm{pc}^{-2}\right]$ & $<10$ & $500-1500$ & 2200 \\
(8) $\mathrm{t}_{\mathrm{GI}}[\mathrm{Myr}]$ & $>10$ & $0.5-1.5$ & 0.5 \\
(9) $\lambda_{\mathrm{J}}[\mathrm{pc}$ & Few $\times(100-1000)$ & $100-300$ & 90 \\
\hline
\end{tabular}

Rows are : (1) $M_{\text {gas }-\mathrm{m}}$, the molecular gas mass (2) $\mathrm{M}_{\mathrm{gas}, \mathrm{m}} / \mathrm{M}_{\mathrm{dyn}}$, the ratio of molecular gas mass to dynamical mass; (3) SFR, the star formation rate; (4) $\Sigma_{\text {gas,m }}$ the surface density; (5) $\sigma$, the velocity dispersion; (6) $\Sigma_{\text {crit }}$, the critical density for the onset of gravitational instabilities (7) $\kappa$, the epicyclic frequency; $(8) t_{G I}=Q / \kappa$, the growth timescale of the most unstable wavelength (9) $\lambda_{\mathrm{J}}$, the Jeans length

Shlosman et al. 1989). The two-fluid disk of gravitationally coupled gas and stars will be more unstable to gravitational instabilities than a purely stellar disk (e.g., Jog 1996). (ii) In the presence of a large epicyclic frequency (several $100-1000 \mathrm{~km} \mathrm{~s}^{-1} \mathrm{kpc}^{-1}$ ) and velocity dispersion (10 to $40 \mathrm{~km} \mathrm{~s}^{-1}$ ), gravitational instabilities can overcome Coriolis and pressure forces only at very high gas densities (few 100-1000 $\mathrm{M}_{\odot} \mathrm{pc}^{-2}$ ). However, once triggered, they grow on a timescale $\left(t_{\mathrm{GI}} \sim\right.$ few Myrs) comparable to the lifetime of an OB star. These conditions can enhance the fraction of gas converted into stars before a molecular cloud is disrupted by massive stars. (iii) Table 1 suggests that the prototypical ultra luminous infrared galaxy (ULIRG) Arp 220 may be a scaled-up version of the starbursts in our sample. ULIRGs may be starbursts which have built an extreme molecular environment (density and linewidths) in the inner few $100 \mathrm{pc}$ of a deep stellar potential well through major mergers or interactions.

\subsection{Circumnuclear Star Formation Morphology and Efficiency}

(i) The starbursts and non-starbursts have circumnuclear SFR of 3-11 and 0.1$2 \mathrm{M}_{\odot} \mathrm{yr}^{-1}$ respectively, from $\mathrm{RC}$ and $\mathrm{Br} \gamma$ data. The starbursts have a larger peak gas surface density $\Sigma_{\text {gas-m }}$ in the inner 500 pc radius than non-starbursts (Fig. 3a). In the starbursts, both $\Sigma_{\text {gas-m }}$ and $\Sigma_{S F R}$ increase towards the inner $500 \mathrm{pc}$ radius (Figs 3a-b). Over the region of intense SF in several starbursts, $\Sigma_{\text {gas-m }}$ remains close to the Toomre (1964) critical density $\left(\Sigma_{\text {crit }}\right)$ for the onset of gravitational instabilities despite an order of magnitude variation in $\Sigma_{\text {crit }}$, as illustrated for NGC 4102 in Figs. 4b-c.

(ii) In the non-starbursts, there are gas-rich regions with no appreciable star formation, for instance, inside the ring of HII regions in NGC 3351 and NGC 4314 , at the CO peaks in NGC 6951, and in the extended gas in NGC 4569 (Fig. 2). The gas surface density, although high, is still sub-critical in regions of inhibited star formation. For instance, in the non-starburst NGC 4314 (Figs 

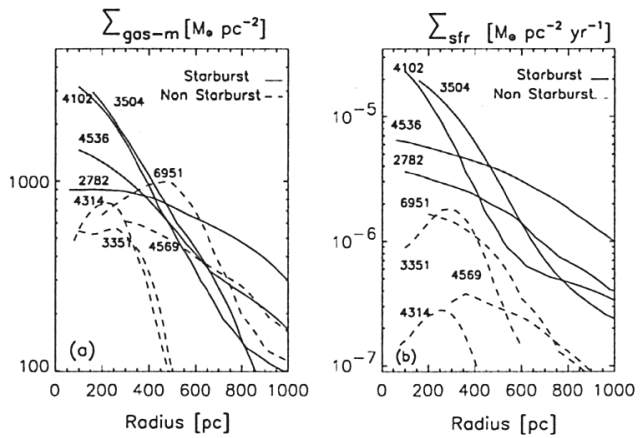

Figure 3. (a) (b) The azimuthally averaged molecular gas surface density $\left(\Sigma_{g a s-m}\right)$ and extinction-corrected SFR per unit area $\left(\Sigma_{S F R}\right)$.
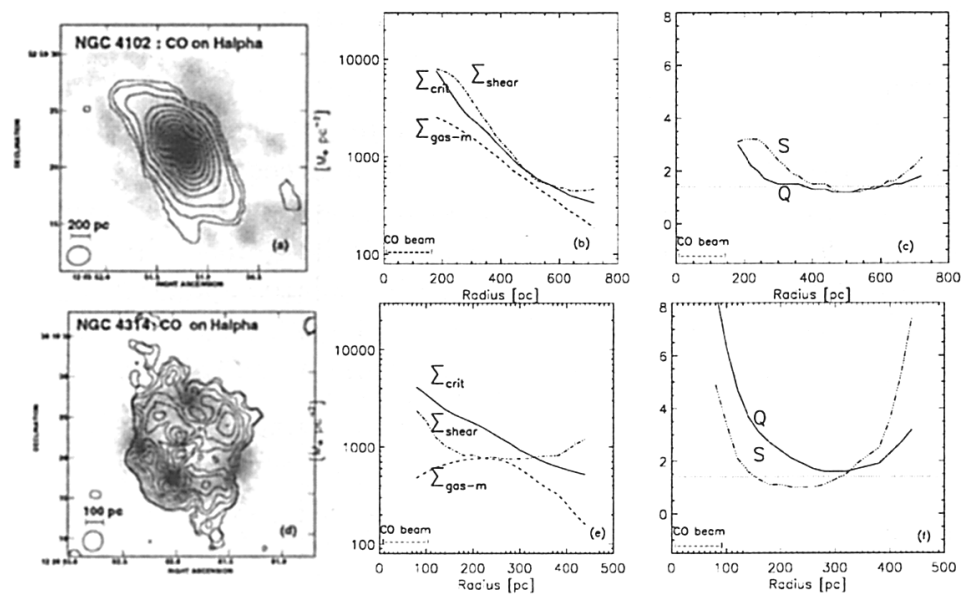

Figure 4. Column 1 shows the $\mathrm{CO}$ distribution (contours) on the $\mathrm{H} \alpha$ (greyscale). Column 2 shows $\Sigma_{\text {gas }-\mathrm{m}}, \Sigma_{\text {crit }}$, and $\Sigma_{\text {shear. Column } 3 \text { shows }}$ the Toomre Q and shear S parameter (Jogee 1999). Quantities are plotted starting at a radius equal to the $\mathrm{CO}$ beam size $\left(\sim 2^{\prime \prime}\right)$.

$4 \mathrm{e}-\mathrm{f}), \mathrm{Q}$ reaches its lowest value (1-2) in the ring of HII regions between $\mathrm{r}=250$ to $400 \mathrm{pc}$, while at lower radii, sub-critical gas densities increase $\mathrm{Q}$ to 6 . In NGC 4569, the large local shear in the extended gas with large non-circular kinematics along the large-scale stellar bar may inhibit star formation. I suggest that circumnuclear starbursts produce a high SFE by developing supercritical surface densities in a large fraction of the gas close to the center, while subcritical densities and large local shear may limit the SFE of non-starbursts.

\section{References}

Jog, C. J. 1996, MNRAS, 278, 209

Jogee, S. 1999, Ph.D. thesis, Yale University

Jogee, S., Kenney, J. D. P., \& Smith, B. J. 1999, ApJ, 526, 665

Shlosman, I., Frank, J., and Begelman, M. C. 1989, Nature, 338, 45

Toomre, A. 1964, ApJ, 139, 1217 